\title{
PLANEAMENTO DA VIDA DE SERVIÇO DE ATIVOS CONSTRUIDOS PARA INCORPORAÇÃO NA METODOLOGIA BIM
}

\author{
Filipa Salvado $^{(1)}$, Álvaro Vale e Azevedo ${ }^{(1)}$, Maria João Falcão Silva ${ }^{(1)}$, Paula Couto ${ }^{(1)}$, \\ Pedro Martins ${ }^{(2)}$
}

(1) Laboratório Nacional de Engenharia Civil (LNEC), Lisboa

(2) Integrated Design Studio (IDS), Leiria

\begin{abstract}
Resumo
O planeamento do ciclo de vida dos ativos construídos fornece a garantia de que a vida útil de um dado ativo, com determinada localização, as suas características próprias e uma manutenção prevista, seja pelo menos tão longa quanto a estimada em projeto. Este plano da vida de serviço dos ativos facilita a tomada de decisões bem fundamentadas, no que se refere a engenharia de valor, planeamento de custos, planeamento de manutenção e impacto ambiental. Dado que se verifica ser complexo estimar com precisão a vida de serviço de ativos construídos, torna-se necessário que se use o conhecimento disponível relacionado com a vida útil de materiais, produtos, componentes e sistemas a serem incorporados nos ativos construídos. Assim, se a vida útil de qualquer um deles for menor que a vida de serviço do ativo estimada em projeto, deve-se tomar uma decisão sobre se a manutenção, a reparação ou a substituição podem garantir que as suas funções essenciais se mantêm de forma adequada. Com vista a apoiar nas especificações e no projeto, bem como evitar situações obsoletas e de desperdício de recursos, o planeamento da vida de serviço dos ativos construídos pode, e deve incluir, entre outras coisas, projeções das necessidades, do tempo de substituição e da recuperação no fim de vida. A norma ISO 15686 está decomposta em várias partes relacionadas com o planeamento da vida de serviço dos ativos construídos, incluindo procedimentos de previsão, utilização de IFC baseadas no BIM, custos do ciclo de vida, avaliação de dados, etc. Assim, o presente artigo pretende, apoiado na norma ISO 15686, contribuir para a otimização e operacionalização do referido planeamento, com o objetivo da sua incorporação na metodologia BIM, sendo identificados e estabelecidos os princípios gerais, a terminologia específica e uma estrutura sistemática, para o planeamento da vida de serviço dos ativos construídos, ao longo do seu ciclo de vida (conceção, estudo prévio, anteprojeto, projeto, construção, operação, manutenção, reabilitação, fim de vida).
\end{abstract}

\section{Introdução}

O planeamento da vida útil de serviço, ou vida de serviço, tem como principal objetivo garantir que a mesma, estimada para um novo ativo construído, num local específico, com manutenção 
planeada e adequada, será pelo menos igual ao previsto na fase de projeto e conceção. Durante a fase de entrega do projeto, para garantir que este atenda aos níveis de requisitos técnicos e funcionais desejáveis, é possível considerar diferentes soluções concetuais para avaliar o impacto de alterações na sua vida de serviço. É assim necessária a realização de uma estimativa adequadamente fiável e robusta da vida de serviço do edifício, utilizando o conhecimento disponível relacionado com a vida de serviço de cada produto, elemento e subsistema a ser instalado no edifício. Se a vida útil de serviço individual estimada para cada elemento ou produto for menor que a vida útil de serviço considerada em projeto, deve ser verificada a possibilidade da manutenção, reparação ou substituição garantirem que as suas funções essenciais são mantidas adequadamente. Para auxiliar na especificação dos produtos na fase de projeto, e evitar obsolescência e desperdício, o planeamento da vida útil de serviço deve incluir projeções futuras sobre custos e momento ótimo para substituições, reparações e recuperação no fim da vida útil (Salvado, Almeida e Azevedo, 2019a). Este planeamento agiliza o processo de tomada de decisão, através da disponibilização de informação relacionada com engenharia de valor, planeamento de custos, planeamento de operação e manutenção, bem como impacto ambiental (Salvado, Almeida e Azevedo, 2018).

A norma internacional ISO 15686, relacionada com o planeamento da vida de serviço, constitui um apoio ao processo de tomada de decisão no que respeita ao desenvolvimento da vida útil do sistema edifício, dos seus subsistemas, elementos e produtos, bem como a outro tipo de ativos físicos. Esta abordagem permite garantir que a vida útil de serviço considerada na fase de projeto tenha uma resposta estruturada ao estabelecer uma base de referência enquadrada numa estimativa de vida útil de serviço. Também garante que o Custo do Ciclo de Vida (CCV) considera fatores ambientais (ex: fim de vida com recuperação de energia incorporada), estando assim o planeamento da vida útil de serviço associado ao desenvolvimento sustentável e ao conceito de valor de toda a vida útil de serviço do ativo.

Existe uma preocupação crescente na sociedade pelos impactos ambientais, económicos e sociais de um ambiente construído. Neste sentido, a utilização de ferramentas de modelação de informação da construção (BIM) surge como uma nova tendência no setor da construção para melhorar as apreciações de carácter sustentável de edifícios na fase de projeto. No entanto, como observado na literatura recente, a falta de informação de carácter semântico nos modelos BIM, pode levar a processos de tomada de decisão ineficazes e modelos inadequados para as fases de operação e de manutenção do ciclo de vida dos edifícios (Santos, Costa, Silvestre e Pyl, 2019).

Atualmente e internacionalmente conhece-se que a incorporação destes conceitos nas práticas correntes do setor da construção é necessária, mas ainda existem alguns desafios na sua implementação de forma a maximizar benefícios económicos e ambientais a longo prazo (Salvado, Almeida e Azevedo, 2018). Neste sentido o presente artigo representa um passo para ultrapassar estes desafios, enquadrando e promovendo a implementação do planeamento da vida útil de serviço de acordo com os requisitos estabelecidos na norma internacional ISO 15686. Interliga-se e explora-se também o potencial dos modelos BIM como um repositório para a informação a utilizar em apreciações do ciclo de vida ao nível económico e ambiental. Assim, este artigo aborda uma temática com elevado interesse e potencial para ser explorada, mas atualmente ainda não existe o desenvolvimento de uma metodologia de aplicação da norma internacional ISO 15686, pelo que se pretende efetuar um levantamento teórico das suas diversas partes, com o intuito de posteriormente ser possível dar continuidade ao muito trabalho ainda por realizar. 
Este estudo identifica o caminho a seguir no que respeita à aplicação dos requisitos da norma ISO 15686 a casos de estudo concretos, representando um primeiro passo e uma base teórica para o desenvolvimento de uma metodologia que permita a incorporação em modelos BIM de informação relativa ao planeamento da vida de serviço de ativos construídos.

\section{Planeamento da vida de serviço: enquadramento normativo}

A norma internacional ISO 15686, para o planeamento da vida de serviço, está em desenvolvimento pelo Subcomité SC14 (Design life) do Comité Técnico ISO/TC 59 (Building construction). Esta norma internacional é constituída por onze Partes e a Figura 1 esquematiza como essas diversas Partes se relacionam entre si e com os tópicos associados.

\section{PLANEAMENTO DA VIDA DE SERVIÇO DE EDIFÍCIOS}

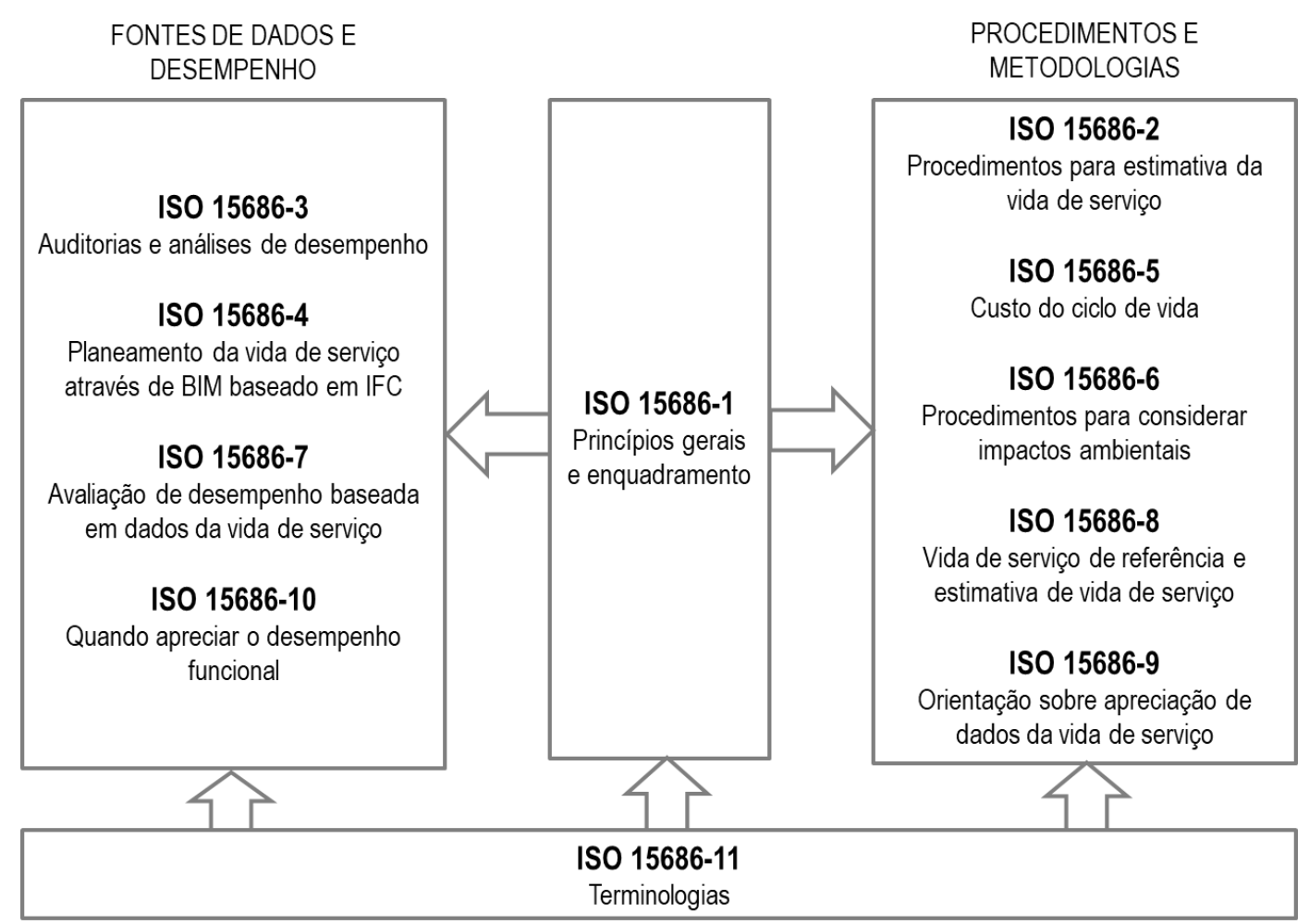

Figura 1: Relações entre as Partes da ISO 15686 e o planeamento da vida útil de serviço de edifícios (adaptado de ISO 15686-1).

Na Figura 2 apresenta-se a relação entre as várias fontes de dados da vida útil de serviço e respetivas abordagens para a estimativa, constantes em diversas Partes da ISO 15686.

Esta norma internacional é relevante para o planeamento da vida útil de serviço de edifícios novos e existentes. Em edifícios existentes, aplica-se principalmente à estimativa da vida útil de serviço residual do sistema edifício, dos seus subsistemas, elementos e produtos que já estão em serviço, à sua seleção e ao detalhe do planeamento de reparações e substituições, bem como de novos trabalhos. Ao se exigir uma estimativa da vida útil de serviço de cada subsistema, 
elemento e produto do edifício, o planeamento da sua vida de serviço auxilia o processo de tomada de decisão sobre especificações e detalhes do projeto. Além disso, podem ser aplicadas técnicas de planeamento de custo e de manutenção do ciclo de vida e engenharia de valor. A fiabilidade e a flexibilidade da utilização do edifício podem ser aumentadas e a probabilidade de obsolescência precoce pode ser assim reduzida.

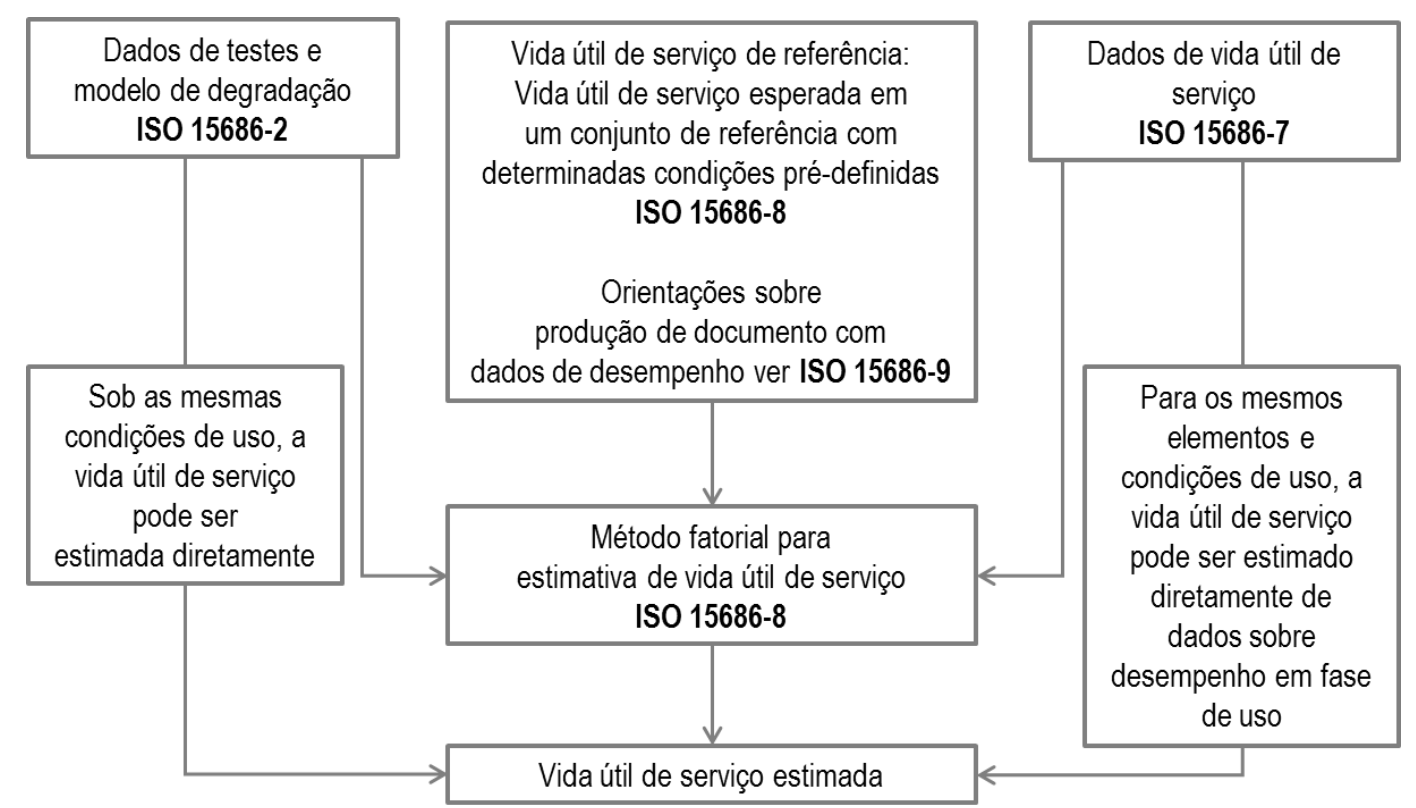

Figura 2: Abordagens para estimativa de vida útil de serviço de edifícios (adaptado de ISO 15686-1)

\subsection{Princípios gerais e enquadramento}

A ISO 15686-1 especifica os princípios gerais do planeamento da vida útil de serviço de um edifício ou outro ativo construído e apresenta uma estrutura sistemática para realizar esse planeamento. Esses princípios gerais também podem ser utilizados para tomar decisões sobre requisitos de operação, manutenção, substituições, reparações e de fim de vida. Esta Parte da ISO 15686 serve também como um guia para as outras Partes.

\subsection{Procedimentos para estimativa da vida de serviço}

A ISO 15686-2 especifica os princípios e procedimentos que facilitam as estimativas da vida útil de serviço dos subsistemas, elementos e produtos do edifício, com base no seu desempenho técnico e funcional. Fornece uma estrutura geral, procedimentos e requisitos para conduzir e relatar esses tipos de estudos, mas não descreve métodos de teste específicos. Também pode ser utilizada como uma lista de verificação para apreciar estudos completos de estimativas de vida útil de serviço. No entanto, não considera a limitação da vida útil de serviço devido a obsolescência ou outros estados de desempenho imprevisíveis ou não mensuráveis.

\subsection{Auditorias e análises de desempenho}

A ISO 15686-3 preocupa-se em garantir a implementação eficaz de auditorias e revisões do planeamento da vida útil de serviço de edifícios. Descreve a abordagem e os procedimentos a serem aplicados ao projeto, construção e, quando necessário, à gestão da utilização e do fim de 
vida de forma a fornecer garantia razoável de que as medidas necessárias para alcançar um desempenho satisfatório ao longo do tempo serão implementadas. As implicações do custo do planeamento da vida útil de serviço e questões mais amplas relacionadas com a sustentabilidade (ex: energia incorporada, ocupação de terreno, etc.) não são desenvolvidas na ISO 15686-3.

\subsection{Planeamento da vida de serviço através de modelo BIM baseado em IFC}

A ISO 15686-4 está em desenvolvimento e pretende descrever os dados necessários para a realização de estimativas da vida útil de serviço. O seu objetivo principal é definir os dados relacionados com a vida de serviço que podem ser necessários em modelos computacionais. A formatação desses dados para inclusão no cálculo dos modelos deve ser apresentada de acordo com a norma internacional ISO 12006.

A ISO 15686-4 pretende também fornecer orientação sobre a estruturação de informação em fontes de dados existentes para permitir a entrega deste conteúdo de informação numa estrutura que esteja em conformidade com os padrões internacionais de troca de informação. O padrão COBie (Construction Operations Building Information Exchange), para a troca de informação em dados tabulares, é utilizado como uma representação alternativa (representação tabular de uma exibição de transferência do esquema IFC - Industry Foundation Classes) de acordo com os requisitos da norma internacional ISO 16739.

A ISO 15686-4 também é aplicável à troca de informação da vida útil de serviço entre categorias de projeto e de software de gestão de informação que possuem interfaces de troca de informação baseadas em padrões, incluindo modelos BIM (Building construction Information Modelling) e CAFM (Computer Aided Facilities Management).

\subsection{Custo do ciclo de vida}

A ISO 15686-5 especifica procedimentos para realizar análises de Custo do Ciclo de Vida (CCV) de edifícios, novos ou existentes, e seus subsistemas, elementos e produtos. Essas análises levam em consideração o fluxo-de-caixa, ou seja, custos e receitas relevantes decorrentes das diversas fases do ciclo de vida (aquisição, construção, utilização e fim de vida). Geralmente inclui uma comparação entre opções ou uma estimativa de custos futuros e pode ser realizada durante um período de análise definido, incluindo um período inferior ao ciclo de vida completo do ativo edifício.

\subsection{Procedimentos para a consideração de impactos ambientais}

A ISO 15686-6 especifica como apreciar, na fase de projeto, os possíveis impactos ambientais de projetos alternativos. Identifica a interface entre a apreciação do ciclo de vida ambiental e o planeamento da vida útil de serviço.

\subsection{Avaliação de desempenho baseada em dados da vida de serviço}

A ISO 15686-7 fornece uma metodologia genérica para uma avaliação do desempenho dos dados da vida útil de serviço de edifícios existentes. Inclui definição dos termos a serem utilizados (orientações sobre as fases de planeamento, respetiva documentação e inspeção e análise e interpretação das avaliações de desempenho) e a descrição de como o desempenho pode ser documentado de forma a garantir consistência. De salientar que, embora o planeamento da manutenção esteja fora do âmbito deste documento, as inspeções orientadas para a manutenção e as subsequentes recomendações podem ter efeitos significativos na vida útil de serviço e no desempenho esperado. 


\subsection{Vida de serviço de referência e estimativa de vida de serviço}

A ISO 15686-8 fornece orientações sobre o fornecimento, seleção e formatação de dados da vida útil de serviço de referência e sobre a aplicação desses dados para fins de cálculo da vida útil de serviço estimada, utilizando o método fatorial. Este documento não fornece orientação sobre como efetuar a estimativa da parte da modificação ou os valores dos fatores A a $\mathrm{G}$, utilizando as condições de uso de referência fornecidas e as condições de uso específicas do objeto.

\subsection{Apreciação de dados da vida de serviço}

A ISO 15686-9 fornece orientação e uma estrutura para derivação e apresentação de dados relacionados com a vida útil de serviço de referência. Em resposta à procura do mercado, os fabricantes podem desenvolver, voluntariamente, declarações de vida útil de serviço para utilização no seu planeamento (em conformidade com as normas ISO 15686-1, ISO 15686-2, ISO 15686-3, ISO 15686-5, ISO 15686-6, ISO 15686-7 e ISO 15686-8).

\subsection{Apreciação do desempenho funcional}

A ISO 15686-10 estabelece quando especificar ou verificar os requisitos de desempenho funcional durante a vida útil de serviço de edifícios e quando verificar a sua capacidade para atender aos requisitos identificados. Para tal, estão definidos procedimentos que estabelecem escalas com níveis de funcionalidade ou avaliam níveis de manutenção e lacunas que possam existir entre a procura e o fornecimento.

\subsection{Terminologia}

A ISO 15686-11 fornece uma compilação dos termos e definições de conceitos normalizados (das diferentes Partes) para estabelecer um vocabulário aplicável aos aspetos da construção e utilização de um edifício (ou obra de engenharia) e o planeamento da sua vida útil de serviço, conforme aplicado nos documentos da ISO/TC59/SC14-Vida útil de projeto. Uma referência cruzada é incluída em cada uma das definições para a Parte específica da ISO 15686 na qual o conceito é definido, bem como para as outras normas internacionais de onde a definição se origina.

\section{Incorporação dos requisitos da ISO 15686 na metodologia BIM}

O desenvolvimento da metodologia BIM tem sido marcado, no passado mais recente, pela sua generalizada adoção enquanto instrumento fundamental para o progresso do sector AECO (Arquitetura, Engenharia, Construção e Operação). Paralelamente a este destaque mais recente, principalmente desde 2016 com a obrigatoriedade do Reino Unido na entrega de determinados projetos públicos em BIM, o referido desenvolvimento apresenta já um importante nível de maturidade adquirido durante o passado mais longínquo, o qual permite a transposição das suas linhas orientadoras para normas internacionais, nomeadamente as normas internacionais ISO. São exemplo desta materialização as normas que se dedicam na totalidade ao BIM, como a já referida norma ISO 16739, assim como as que se dedicam apenas em parte, como a norma ISO 15686, que analisamos no presente artigo. Com base neste contexto, o presente capítulo será dedicado a detalhar como estão estabelecidas as relações entre o tema do custo do ciclo de vida e a metodologia BIM. 
Como já referido, o principal objetivo da ISO 15686-4:2014 é definir os dados (leia-se informação) relacionados com a vida de serviço que possam ser integrados em processos computacionais para obter resultados, de forma manual ou automatizada, que apoiem a gestão dos ativos durante os seus respetivos ciclos de vida. Por este motivo, a norma define que é importante criar um sistema de gestão da informação assente no cumprimento dos seguintes critérios:

1. Obtenção de informação, assim como suporte de métodos, em quantidade suficiente que permitam calcular os efeitos do meio ambiente sobre os componentes e os materiais utilizados na construção;

2. Definição de planos de manutenção para diferentes qualidades de materiais instaladas em diferentes condições ambientes da envolvente;

3. Aplicação das metodologias de cálculo dos custos no ciclo de vida, com base na informação obtida, para comparação dos benefícios entre soluções com materiais de elevada performance (de reduzida manutenção) e materiais de baixa performance (de maior manutenção), tendo em consideração a facilidade de substituição e demolição;

4. Incorporação do conhecimento e metodologias preditivas para avaliação da performance dos materiais sem que sejam afetadas as metodologias vigentes e a informação já estruturada que permitem o cálculo com base no conhecimento atual;

5. Suporte da interoperabilidade entre diferentes sistemas informáticos;

6. Orientação do seu uso, durantes as diferentes fases do ciclo de vida da construção, por parte de projetistas, construtoras, proprietários, entidades exploradoras e entidades responsáveis pela demolição.

Tendo em consideração que a norma internacional ISO 16739 baseia o seu foco na produção de informação em linguagem EXPRESS, considerada aqui como linguagem de programação, manter-se-á parte da terminologia própria em língua inglesa por corresponder à exigência da referida norma.

\subsection{Princípios gerais e enquadramento}

O planeamento da vida útil de serviço caracteriza-se pela utilização de informação relativa a todos os elementos que constituem os ativos construídos. O uso de tal informação influencia não apenas a fase de projeto como também a fase de utilização e manutenção através do carácter preditivo e estimativo de vida de serviço. A presente norma, suportada no BIM enquanto metodologia para gerir e modelar a informação da construção, estabelece um conjunto de conceitos para serem incorporados todos os dados relativos à vida de serviço. Os objetos BIM são, por este motivo, o principal elemento de projeto enquanto contentor de informação. Nesse sentido, a informação necessita ser estruturada a fim de poder ser partilhada entre todos os intervenientes nas diferentes fases do ciclo de vida, ou seja, tanto de projeto como de construção e utilização. Por este motivo, o suporte no formato IFC torna-se fundamental para a partilha de informação entre todos os intervenientes, de forma interoperável. A partilha de informação deverá seguir uma estruturação padronizada cujos requisitos estejam claramente definidos de maneira a serem seguidos procedimentos normalizados. 
Tabela 1: Conceitos IFC relevantes para a vida útil de serviço e avaliação de impactos (traduzido de ISO 15686-4)

\section{Conceitos na estrutura IFC}

Vida útil

Fatores da vida útil

\section{Objetivo}

Pode ser aplicado a qualquer objeto físico como uma ocorrência única ou uma agregação ou montagem de objetos físicos que atuam como um único objeto. Uma vida útil pode ter um ou mais fatores de vida útil relacionados, de acordo com o método usado pela ISO 15686.

O termo "objeto físico" é usado no presente contexto para identificar a diferença entre um objeto que possui existência física e não um objeto abstrato, como custo ou restrição.

Material Uma definição de material pode estar relacionada a um objeto físico.

Impacto Um ou muitos impactos económicos ou ambientais podem ser associados a objetos físicos de produtos ou processos. Os impactos estão associados a estádios específicos do ciclo de vida.

Condição

A condição atual dos objetos físicos pode ser determinada aplicando um ou mais critérios de condição. A condição pode ser determinada usando avaliação subjetiva (por exemplo, condição em uma escala de 1 a 10 onde 10 é bom e 1 é ruim) ou por avaliação objetiva usando valores medidos.

Conjuntos de quantidades (Quantity sets)

O IFC tem a capacidade de associar quantidades medidas (por exemplo, medidas de contagem, distância ou peso) a um objeto em que não é possível medir essa quantidade a partir da representação usada ou existem regras nacionais específicas que precisam ser aplicadas para a medição de quantidade

Conjuntos de propriedades (Property sets)
Propriedades são atributos adicionais que podem ser definidos e capturados num modelo IFC. As propriedades são geralmente agrupadas em coleções nomeadas, conhecidas como conjuntos de propriedades. Os conjuntos de propriedades podem ser usados como base para armazenar dados externos ou para fornecer dados de uma fonte de dados externa.

Adicionalmente, a presente norma encontra no formato COBie uma forma complementar para, de maneira standard, partilhar informação com uma representação em folha de cálculo tipo Excel.

\subsection{Procedimentos para partilha de dados}

A norma 15686-4:2014 define um mapeamento para o processo de partilha de informação bem como o contexto em que tal informação teve ser partilhada, identificando os critérios de envio 
e receção. Tal mapeamento deriva da ISO 15686-1, nomeadamente o anexo B, bem como do plano de gestão dado pela ISO 15686-3. Em suma o processo geral para partilha de dados sobre a vida de serviço pode ser subdividido em quatro estádios:

1. A Fase de Teste, na qual os produtos passam a ter indicadas as características correspondentes da vida de serviço;

2. A Fase Preditiva, na qual as características definidas no estádio anterior são enquadradas num contexto mais específico, de maneira a ser prevista uma vida de serviço;

3. A Fase de Custo, na qual são adicionadas informações relativas a custos e/ou impactos ambientais que têm influência no valor final a calcular;

4. A Fase da Inspeção em Utilização, a qual permite, de maneira iterativa, reavaliar as características definidas nos estádios anteriores para maior afinação dos resultados futuros.

A determinação da vida útil é realizada em diferentes momentos do projeto, da construção e da operação de um ativo construído. Durante as fases iniciais do projeto, quando as informações do produto são adicionadas, é apenas a vida de um produto que pode ser determinada. Nas fases posteriores do projeto, quando produtos individuais são localizados e esses produtos são designados por tipo, a vida útil do projeto pode ser indicada para todas as ocorrências no nível do tipo. Da mesma forma, quando produtos individuais são identificados, torna-se possível determinar uma vida útil de referência quando um fabricante / fornecedor pode ser também identificado. Assim como na vida útil do projeto, a vida útil de referência pode ser alocada ao nível do tipo de produto. Nas fases posteriores do projeto e durante a construção, quando a configuração e a localização dos produtos estiverem totalmente estabelecidas, torna-se possível analisar a vida útil dos produtos de acordo com as condições de utilização. Essas condições podem variar a vida útil de referência, dependendo de fatores como exposição à intempérie, agressividade do ambiente local e outros fatores que possam alterar a velocidade de degradação. Fruto da aplicação das condições de utilização, é possível definir uma vida útil estimada cujo período é idêntico ao de um ciclo de vida de ocorrência do produto.

\subsection{Requisitos de estruturação de dados}

A identificação de um produto é vital para a troca efetiva das informações. As informações devem ser fornecidas para:

1. o próprio produto, inicialmente como um tipo de biblioteca abstrata e, posteriormente, como uma ocorrência instanciada;

2. a identidade do produto, incluindo a sua designação e nomenclatura, descrição e outras propriedades que o identificam de maneira única;

3. a fonte do produto, em termos das organizações de origem, autor e quaisquer documentos de referência;

4. opcionalmente, classificação e agrupamento do produto de acordo com a prática local.

Os produtos são representados em resumo pelos subtipos de IfcElementType e em uso pelos subtipos de IfcElement. Cada um deles possui atributos que permitem a seleção de enumerações predefinidas e texto livre para definir melhor a natureza do produto. Geralmente, o IfcElementType será desenvolvido com recurso a um atributo PredefinedType. Especificação adicional poderá ser dada na propriedade ObjectType do IfcElement. Uma ocorrência de um produto num projeto usa um subtipo de IfcElement, tendo um posicionamento no espaço $2 \mathrm{D}$ ou 
3D e, pelo menos, uma representação de forma. Importa adicionar à informação associada aos produtos, também, um histórico do proprietário com uma definição do autor. Uma ou mais classificações podem ser associadas a um produto indicando propriedades como o nome, fonte e edição do sistema de classificação

O agrupamento de propriedades definidos pelos conjuntos referidos (Property Sets) têm conjuntos já definidos e procedimentos específicos que, pela sua extensão, não serão reproduzidos no presente artigo. Importará referir que as referidas propriedades são, no presente momento, o conjunto mínimo para que os dados possam ser devidamente interpretados. De acordo com o recente artigo publicado sobre a relação Análise de Ciclo de Vida e Custo no Ciclo de Vida (Santos, Costa, Silvestre e Pyl, 2019) é possível estabelecer uma forte relação entre propriedades comuns, as quais utilizarão os mesmos conceitos IFC. No entanto, os autores referem que as propriedades já contidas no IFC apenas permitem uma avaliação simplificada e que, para análise do ciclo de vida completo de um projeto em ambiente BIM, propriedades adicionais terão de ser introduzidas.

\section{Conclusões}

O processo de maturidade do BIM tem sido conseguido não apenas pela criação de normalização específica como, também, pela atualização de normalização existente. A norma internacional ISO 15686 é exemplo do processo de atualização referido, com a incorporação de uma das suas partes totalmente dedicada ao BIM. Decorrente da necessidade de partilha de informação, a norma baseia-se no formato IFC que continua pouco conhecido e, principalmente dominado, pela comunidade de intervenientes que se suportam do BIM. Por este motivo, a existência de software específico para análise do Custo no Ciclo de Vida, maioritariamente integrada na análise mais abrangente de Avaliação do Ciclo de Vida, tem sido o caminho encontrado para realizar os estudos atuais.

A vantagem da presente norma se fundamentar no IFC é a de permitir que qualquer utilizador BIM, ou até as proprietárias de software, possam criar os conjuntos de propriedades nos modelos produzidos e, com isso, facilitar a exportação dos dados. A sua desvantagem poderá residir no facto de ser constituída por diversas partes (11 no total), o que obriga a um investimento financeiro significativo para se conseguir obter uma visão abrangente, adicionado ao fato de que as suas revisões não ocorrem em simultâneo, o que obriga a um acompanhamento mais próximo da totalidade da norma.

Dada a reduzida bibliografia sobre o tema, principalmente no panorama nacional, o presente artigo pretende marcar, para os autores, o início de uma investigação mais aprofundada sobre o planeamento da vida de serviço de ativos construídos na metodologia BIM, com o objetivo de poder materializar e apresentar os conceitos e processos indicados.

Salienta-se que já têm vindo a ser desenvolvidos alguns casos de estudo relacionados com esta temática (Santos, Costa e Silvestre, 2019; João, 2018), mas a plena aplicação deste tipo de metodologias requer a existência de informação robusta e fiável disponível em bases de dados acessíveis aos intervenientes no processo (Salvado, Almeida e Azevedo, 2019b).

Outras aplicações a casos de estudo exemplificativos, que contribuirão para a melhor compreensão do tema, encontram-se em desenvolvimento pelos autores e serão objeto de futuras publicações. Salienta-se ainda que diversos grupos de trabalho têm vindo a ser constituídos recentemente para desenvolver estudos nesta área (LDAC; WBDG; W3C). 


\section{Referências}

[1] ISO 12006-2:2015 - Building construction - Organization of information about construction works - Part 2: Framework for classification. International Organization for Standardization.

[2] ISO 12006-3:2007 - Building construction - Organization of information about construction works - Part 3: Framework for object-oriented information. International Organization for Standardization.

[3] ISO 15686-1:2011 - Buildings and constructed assets - Service life planning - Part 1: General principles and framework. International Organization for Standardization.

[4] ISO 15686-2:2012 - Buildings and constructed assets - Service life planning - Part 2: Service life prediction procedures. International Organization for Standardization.

[5] ISO 15686-3:2002 - Buildings and constructed assets - Service life planning - Part 3: Performance audits and reviews. International Organization for Standardization.

[6] ISO/TR 15686-4:2014 - Buildings and constructed assets - Service life planning - Part 4: Service Life Planning using IFC based Building Information Modelling. International Organization for Standardization.

[7] ISO 15686-5:2017 - Buildings and constructed assets - Service life planning - Part 5: Lifecycle costing. International Organization for Standardization.

[8] ISO 15686-6:2004 - Buildings and constructed assets - Service life planning - Part 6: Procedures for considering environmental impacts. International Organization for Standardization.

[9] ISO 15686-7:2017 - Buildings and constructed assets - Service life planning - Part 7: Performance evaluation for feedback of service life data from practice. International Organization for Standardization.

[10]ISO 15686-8:2008 - Buildings and constructed assets - Service life planning - Part 8: Reference service life and service-life estimation. International Organization for Standardization.

[11]ISO/TS 15686-9:2008 - Buildings and constructed assets - Service life planning - Part 9: Guidance on assessment of service-life data. International Organization for Standardization.

[12]ISO 15686-10:2010 - Buildings and constructed assets - Service life planning - Part 10: When to assess functional performance. International Organization for Standardization.

[13]ISO/TR 15686-11:2014 - Buildings and constructed assets - Service life planning - Part 11: Terminology. International Organization for Standardization.

[14]ISO 16739-1:2018 - Industry Foundation Classes (IFC) for data sharing in the construction and facility management industries - Part 1: Data schema. International Organization for Standardization.

[15]João, S. (2018). Metodologia BIM Aplicada a Gestão de Ativos. Dissertação de Mestrado em Engenharia Civil, Instituto Superior de Engenharia de Lisboa (ISEL).

[16] Salvado, F.; Almeida, N.; Vale E Azevedo, A. (2018). Towards improved LCC-informed decisions in building management. Journal of Built Environment Project and Asset Management, Vol. 8:2, pp.114-133.

[17] Salvado, F.; Almeida, N.; Vale E Azevedo, A. (2019a). Aligning financial and functional equivalent depreciations rates of buildings assets. Engineering, Construction and Architectural Management. 
[18]Salvado, F.; Almeida, N.; Vale e Azevedo, A. (2019b). Economic information networks for improved lifecycle management of constructed assets. WCEAM2019 - World Congress on Resilience, Reliability and Asset Management, Singapura, 28-31 julho.

[19] Santos, R.; Costa, A.; Silvestre, J. \& Pyl, L. (2019). Integration of LCA and LCC analysis within a BIM-based environment. Automation in Construction, Vol.103, pp.127-149.

[20]LDAC - Linked Data in Architecture and Construction http://linkedbuildingdata.net/ldac2020/ (acedido a 01/07/2020).

[21] WBDG - Whole Building Design Guide - https://www.wbdg.org/ (acedido a 01/07/2020).

[22]W3C Community and Business Groups - Linked Building Data Community Group https://www.w3.org/community/lbd/ (acedido a 01/07/2020). 